\title{
The Effect of Non-Native Accents and Stereotypes on Speaker Perception and Comprehension
}

\author{
Susanna Baghdasaryan \\ Kh. Abovyan Armenian State Pedagogical University
}

\begin{abstract}
The paper argues that linguistic forms per se do not have any positive or negative meaning and that one form is not better than the other. However, this is not fully perceived outside the narrow linguistic community. Very often it is demonstrated by the apparent stigmatization of non-native accents by native speakers of the language. This phenomenon seems true in reference to many communities and languages. Different sociolinguists have conducted research to prove the ungrounded nature of linguistic partiality. They try to account for the listeners' attitudes towards non-native speech by incorporating the model of dual cognitive processing methods, namely implicit and explicit. They believe that attitudes are formed by the interaction of these two cognitive mechanisms. The negative attitude can be cured by application of more explicit cognitive techniques.
\end{abstract}

Key words: native speakers, non-native accents, Sociolinguistics, cognitive mechanisms, partiality, implicit/explicit mechanisms, stigmatization.

\section{Introduction}

There is a wide-spread belief that language is one of the major uniting factors for any society. However, the multiplicity of one and the same language in a given community may be viewed as simply being incompatible with a homogenous healthy society. Moreover, it should be mentioned that there is a pretty high level of intolerance towards other regional language varieties spoken by minorities. This phenomenon seems true in reference to many communities and languages. The scientists within the field of Sociology, 
Sociolinguistics and Psychology have tried to give a rational explanation about the causes of non-native or regional accent stigmatization. The brief literature review that has been carried out for this research shows that such studies have been more actively conducted in the US than anywhere else in the world. It is not because the non-native accent stigmatization is not ubiquitous but simply because this phenomenon seems to be more painful for the US society by merely contradicting the philosophy on the basis of which this country was created.

One of the varieties of English, is termed as American English. Any other variety is considered non-standard or accented. Even certain US regional language varieties are looked down upon, let alone accents which are associated with foreignness and belong to immigrants.

All the researchers seem to agree on the fact that non-native accent stigmatization is associated with prejudiced beliefs about cultures that these non-native speakers represent. We will start off by presenting a few studies on this topic. Then we will proceed with researches that analyze concrete cases of bias towards a certain accent and will provide a brief review of the experiments that different sociolinguists have conducted to prove the ungrounded nature of linguistic partiality.

\section{Cultural Bias as a Determiner of Unfavorable Attitudes towards}

\section{Non-Native Accents ${ }^{1}$}

Julie Spencer-Rodgers and Timothy McGovern (2002:610) have conducted a very important research with the aim of trying to explain some objective factors that underlie the cultural bias. They point out that intercultural communication difficulties arise from:

- Group differences in cognition (e.g. values, norms, etc.);

- Affect (e.g. types and levels of emotional expressivity);

- Patterns of behavior (e.g. language customs, communications styles, etc.

The authors believe that individuals who are involved in intercultural communication face language barrier challenges - challenges that arise from the difference in cultural beliefs. These are serious tasks which can have adverse 
emotional consequences both for foreigners (or immigrants) and for the socalled host groups.

The authors do not try to justify any type of cultural prejudice; they try to find the objective causes that might underlie this ungrounded belief and possible ways of overcoming them.

The participants of the research are undergraduate students (both native speakers and non-native English speakers/international students) in a US educational institution.

Before presenting the experiment, it is important to mention that mentioned authors consider the following main factors that cause prejudice: negative stereotypes, intergroup anxiety, realistic threats, and symbolic/cultural threats (Spencer-Rodgers \& McGovern 2002:613).

The authors designed questionnaires which would measure the abovementioned four factors. Their initial hypothesis was that "intercultural communication emotions would constitute a unique and potent source of attitudes toward ethnolinguistic outgroups" (ibid 614).

The researchers have concluded that an affective factor is a determining factor in forming attitudes towards foreign students. As the authors point out, "many American college students, have difficulties with an international student on their campuses. Factors such as accented speech, cultural differences in non-verbal communication styles, and cultural variations in values, norms and customs contribute to these communication problems" (ibid 623-624).

While we agree with the authors that affective factors, the awkwardness arising from the difficulty of dealing with unfamiliar cultures, and the cognitive difficulty of processing an accented speech may play a role in formation of a cultural bias, the persuasion of his/her own cultural superiority and ungrounded beliefs are certainly a crucial factor determining the unfavorable attitudes towards other cultures, hence towards non-native accents associated with the representatives of those cultures.

In her article Listening with Attitude published in 2010, S. Lindemann describes an experiment during which two groups of participants were asked to listen to a recorded lecture which was facilitated by a photo of an Asian for the 
first group and by that of a Caucasian for the second one. The native speakers in the second group reported that they perceived a milder accent than the ones in the first group although both groups were listening to the same voice (Lindemann 2010:420).

Another important research that should be mentioned when talking about the cultural attitudes is the article by A. Pantos and A. Perkins. The authors try to account for the listeners' attitudes towards non-native speech by incorporating the model of dual cognitive processing mechanisms, namely implicit and explicit. The interaction of these two cognitive mechanisms are believed to form these attitudes. Implicit attitudes are the person's immediate, automatic reactions based on preexisting stereotypes and cognitive connections, and explicit attitudes are reactions formed through additional controlled cognitive processing. Pantos and Perkins (2013:5) state that implicit attitudes, per se, are not accessible for consciousness, therefore they cannot be studies by applying self-reflective methods. Such attitudes can only be investigated by using indirect methods which do not rely on human introspection. The implicit - explicit distinction is directly linked to the idea of in-group vs. out-group dichotomy which, according to authors of this article, underlies the unfavorable attitudes towards non-native accents. They believe that non-native speech associated with being out of group which once perceived, implicitly provokes a negative attitude. The application of more explicit cognitive mechanisms will help to make objective judgments and to avoid instinctive and logically unsupported reactions.

\section{The Non-Native Accent As a Marker of Inferior Cognitive Capacities}

Sometimes, even subconsciously, the native speakers of the language attribute inferior cognitive capacities to non-native speakers. Because of these misconceptions, some native speakers fail to play their role in the process of communication. In other words, they simply refuse to cooperate. Conversation is not a unilateral process: it requires some cooperation from all the parties. When one or more of the parties fails to cooperate, the communication becomes impossible even among native speakers. 
Precisely this idea is elaborated in the article Listening with an attitude by S. Lindemann published in 2002. The author hypothesizes and later proves with an experiment that the attitude of the listener towards the non-native speech is extremely important for its comprehension.

As a part of the experiment, a group of native-speakers was chosen. The group consisted of members who had positive attitudes towards Koreans and Korean accent, and of those whose attitudes are not that positive and are sometimes even negative. The native speakers of Korean were supposed to explain to the native English speakers how to draw a certain missing route on an imaginary map. The results of the study showed that those participants who had positive attitudes towards Koreans and Korean accent, understood the explanations given by native Korean speakers, while the participants who had more negative attitudes claimed that they did not understand the foreign accent.

This study is a clear demonstration of the fact that willingness to participate in a conversation is a key to a successful communication.

In her article, Lindemann frequently quotes the famous sociolinguist Rosina Lippi-Green, namely from the latter's article Language ideology and language prejudice (2004), where Lippi-Green talks about the myth of ideal standard English. The author elucidates the process of generation of this idealized language form. Her statement is so clear and to the point that we cannot help but directly quote it: "Dominant institutions promote the notion of overarching, homogenous standard language. That language is primarily white, upper-middle class and middle American: it is often claimed to be unaccented. But of course it is accented, like all other language verities. It just happens to be the accent of the mainstream" (Lippi-Green 2004:294).

In this context, it is worth recalling another famous article by Lindermann, titled Who is unintelligible (2010). In this article the author refers to the famous policy of the state of Arizona about English teachers with non-native accent not having the right of teaching English at US educational institutions. Lindermann (2010:224) points out that the very idea that English teachers should not have an accent is absurd simply because everyone has an accent. 
All the studies reviewed above are more concentrated on the perception of the listener. The impact of the non-native accent on the speaker has not been studied much. The research by A. Gluszek and F. Dovidio (2010) is one of the first studies that mainly concentrates on the speaker's experience. The authors hypothesize that non-native accent can create a negative experience for the speaker. The expectation that their accent will be stigmatized facilitates the feeling of not belonging to the US. Gluszek and Dovidio conducted two studies to prove or disprove their hypothesis. The first study tested the association between having a nonnative accent and perceptions of discrimination and experiences in communication. The second study was aimed at investigating the link between having a non-native accent as compared with having regional non-standard accent and feeling of belonging (Gluszek and Davidio 2010:225). The participants in the study 1 were supposed to complete an online survey. They were to answer the following questions: Are they native speakers? What is their level of Education? They also had to assess their personal experience of bias. Study 2 included a comparison between responses of people with nonnative accents and those with regional accents to help identify the effects of having non-native accent rather than a regional accent.

The researchers concluded that the participants with nonnative accents perceived more problems in communication than the ones with regional accents. In addition, the participants with non-native accents had a lower sense of belonging to the US than the ones with regional non-standard accents (Gluszek \& Dovidio 2010:226).

As it has been mentioned at the beginning of this subsection, non-native accents are sometimes wrongly associated with inferior cognitive capacities and can have a huge effect on the degree of perceived credibility of the speaker. This problem has been analyzed by Shiri Lev-Ari and Boaz Keysar from the University of Chicago. The article that encompasses this up-mentioned analysis is entitled Why do not we believe non-native speakers? (2010).

Lev-Ari and Keysar try to analyze the impact of accented speech on the credibility if the message from an objective point of view. That is to say, they do not simply claim that this misconception is a result of prejudiced beliefs but 
rather, they try to explain why such an attitude exists. In fact, they believe that this perception has two aspects: one of them is that accent serves as a signal which prompts the out of group perception of the non-native speaker. According to the authors, this perception creates a biased perception of untruthfulness. The second aspect is that the accented speech is harder to process and this higher processing cost creates a perception of untruthfulness of the statement. In other words, the authors claim that "[...] the difficulty of understanding accented speech has a unique effect on perceived credibility which cannot be attributed to stereotypes about non-native spekers" (Lev-Ari \& Keysar 2010:1093).

Levi-Ari and Keysar conducted two experiments to test their hypothesis. In the first experiment the native speakers were asked to judge trivial statements pronounced by native speakers and non-native speakers with different accents. In Experiment 2 the authors simply revealed the purpose of their study to the participants. They believed that if the listeners were aware that the difficulty of processing non-native accents incorrectly influences their credibility judgments, they would review their attitudes.

The first experiment proved that the statements pronounced with nonnative accents have a lower credibility rate. The second experiment proved that many native speakers review their credibility judgments when they are cautioned against associating an accent with the idea of truthfulness. Thus, the authors concluded that the idea that some non-native accents have a higher processing cost on behalf of the native speakers is objectively real. But what is truly wrong and should be eliminated is the idea of associating the difficulty of processing a non-native accent with credibility in the speaker's message (LevAri \& Keysar 2010:1095).

\section{Conclusion}

It is understandable that if a certain language community is used to a certain accent, they are not always ready to accept another variety not only because of a bias towards the cultural identity of the non-native speaker but simply because it is different. Some native speakers even question why the non-native 
speakers cannot try and reach a native fluency. The fact is that it is almost impossible to gain a native fluency in any language after a certain age. This phonological barrier, however, does not have anything to do with other human cognitive systems. In other words, a non-native accent should never be associated with lack of intelligence. These concepts are recognized in linguistic circles but they are also limited to these circles. Perhaps, one way of preventing biased attitudes towards different non-native accents will be the incorporation of basic linguistic courses in high school curriculums. This kind of education is of vital importance for multicultural societies.

Another important factor that should be recognized by both native and nonnative speakers is that communication is not a unilateral process: both sides should make an equal effort in assuring an efficient conversation. If any of the sides fails to carry out the conversational burden, the communication will fail even if the speakers are both speakers of the same language variety.

Humans are biological systems and human language is a biological module. There are no better or worse language varieties because all human languages conform to a universal innate structure. Different language varieties are just different forms of expressing one and the same innate structure. There are no superior or inferior sound forms, hence, there cannot be any superior or inferior accent - they are just different.

\section{Notes:}

1. Non-native speech is not associated exclusively with the phonological characteristics as the word accent might suggest; it certainly has other very important morpho-syntactic components. However, for the purpose of this research we will refer mainly to the phonological aspect of non-native speech. 


\section{References:}

1. Adank, P.; Evans, B.; Stuart-Smith, J. \& Scott, S. (2009) Comprehension of Familiar and Unfamiliar Native Accents under Adverse Listening Conditions. // Journal of Experimental Psychology: Human Perception and Performance, 35(2), pp. 520-529.

2. (1987) Ethnicity and Language. WI, Milwaukee: University of Wisconsin System, Institute on Race and Ethnicity.

3. Gluszek, A. \& Dovidio, J. (2010) Speaking with a Nonnative Accent: Perceptions of Bias, Communication Difficulties, and Belonging in the United States. // Journal of Language and Social Psychology, 29(2), pp. 224234.

4. Hansen, J. \& Ikeno, A. (2007) The Effect of Listener Accent Background on Accent Perception and Comprehension.// EURASIP Journal on Audio Speech and Music Processing, (1).

5. Ikeno, A. \& Hansen, J. (2007) The Effect of Listener Accent Background on Accent Perception and Comprehension.// EURASIP Journal on Audio Speech and Music Processing, (1).

6. Lev-Ari, S. \& Keysar, B. (2010) Why don't We Believe Non-Native Speakers? The Influence of Accent on Credibility. // Journal of Experimental Social Psychology, 46(6), pp. 1093-1096.

7. Lindemann, S. (2002) Listening with an Attitude.// Language in Society, Vol. 31, No. 3, pp. 419-441.

8. Lindemann, S. (2010) Who's “Unintelligible"? The Perceiver's Role. // Issues in Applied Linguistics, 18(2), pp. 223-232.

9. Lippi-Green, Rosina (2016) Language Ideology and Language Prejudice.// Language in the USA. Cambridge: CUP, pp. 289-304.

10. Pantos, A. \& Perkins, A. (2013) Measuring Implicit and Explicit Attitudes toward Foreign Accented Speech. // Journal of Language and Social Psychology, 32(1), pp. 3-20.

11. Spencer-Rodgers, J. \& McGovern, T. (2002) Attitudes toward the Culturally Different: the Role of Intercultural Communication Barriers, Affective 
Responses, Consensual Stereotypes, and Perceived Threat. // International Journal of Intercultural Relations, 26(6), pp. 609-631.

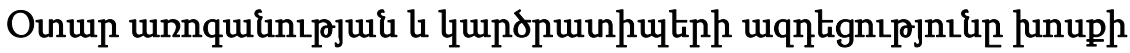
piquulưư પnu

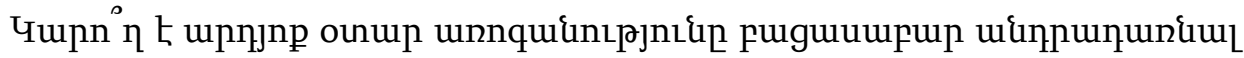

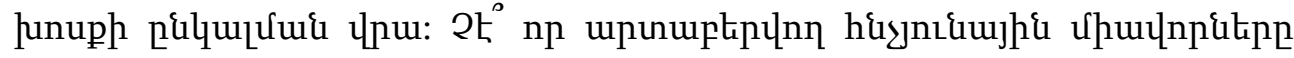

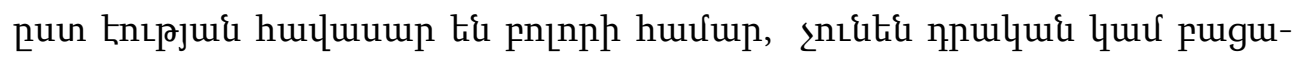

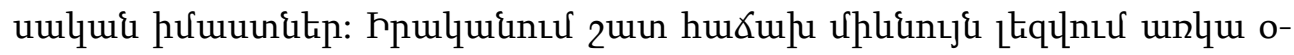

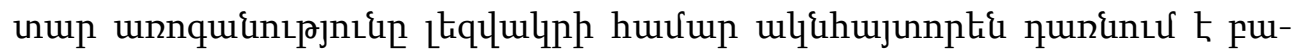

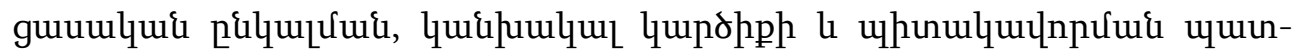

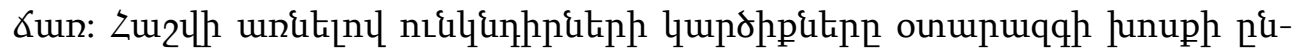

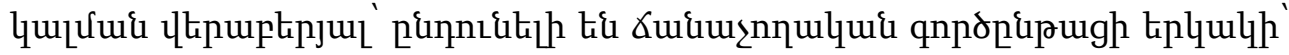

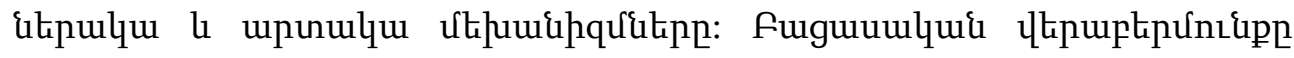

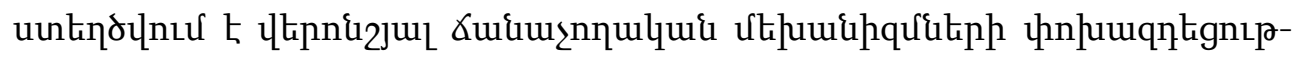

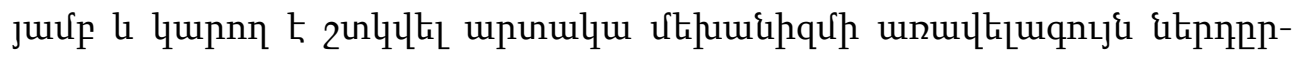
ưưp: 